\title{
Carbon irradiation overcomes glioma radioresistance by eradicating stem cells and forming an antiangiogenic and immunopermissive niche
}

\author{
Sara Chiblak, ${ }^{1,2}$ Zili Tang, ${ }^{1,2}$ Dieter Lemke, ${ }^{1,3}$ Maximilian Knoll, ${ }^{1,2}$ Ivana Dokic, ${ }^{1,2}$ Rolf Warta, ${ }^{1,4}$ \\ Mahmoud Moustafa, ${ }^{1,2}$ Walter Mier, ${ }^{1,5}$ Stephan Brons, ${ }^{2}$ Carmen Rapp, ${ }^{1,4}$ Stefan Muschal, ${ }^{1,2}$ \\ Philipp Seidel, ${ }^{1,2}$ Martin Bendszus, ${ }^{1,3}$ Sebastian Adeberg, ${ }^{1,2}$ Otmar D. Wiestler, ${ }^{1}$ Uwe Haberkorn, ${ }^{1,5}$ \\ Jürgen Debus, ${ }^{1,2}$ Christel Herold-Mende, ${ }^{1,4}$ Wolfgang Wick, ${ }^{1,3}$ and Amir Abdollahi',2 \\ ${ }^{1}$ Cerman Cancer Consortium, Heidelberg, Germany. ${ }^{2}$ Division of Molecular \& Translational Radiation Oncology, Heidelberg \\ Ion Therapy Center (HIT), Heidelberg Institute of Radiation Oncology, Heidelberg University Medical School and National \\ Center for Tumor Diseases, German Cancer Research Center, Heidelberg, Germany. ${ }^{3}$ Department of Neurology, Heidelberg \\ University Medical School and National Center for Tumor Diseases, German Cancer Research Center, Heidelberg, Germany. \\ ${ }^{4}$ Division of Experimental Neurosurgery, Department of Neurosurgery, University of Heidelberg Medical School, Heidelberg, \\ Germany. ${ }^{5}$ Department of Nuclear Medicine, Heidelberg University Medical School and National Center for Tumor Diseases \\ German Cancer Research Center, Heidelberg, Germany.
}

Tumor radioresistance leading to local therapy failure remains a major obstacle for successful treatment of high-grade glioma. We hypothesized that distinct radiobiological features of particle therapy with carbon ions may circumvent glioma radioresistance. We demonstrate that carbon irradiation (CIR) efficiently eradicates radioresistant patient-derived glioma stem cells (CSCs), leading to growth inhibition and prolonged survival. The impact of CIR at the tumor-stroma interface was further investigated in 2 syngeneic mouse and 2 orthotopic CSC xenograft models. Intriguingly, tumor regressions and long-term local controls were observed at doses greater than or equal to 15-Gy CIR. Fractionated CIR further prolonged survival. The enhanced relative biological effectiveness of CIR in vivo was attributed to its potent antiangiogenic effects and eradication of radioresistant hypoxic tumor cells. Blockade of the HIF1- $\alpha /$ stromal cell-derived factor 1 / CXCR4 axis by CIR reduced the recruitment of microglia and myeloid-derived suppressor cells $\left(\mathrm{CD} 11 \mathrm{~b}^{+} \mathrm{Gr}^{+}\right)$. Consequently, CIR abrogated M2-like immune polarization and enhanced the influx of CD8 ${ }^{+}$cells, generating an immunopermissive niche. We report that radiotherapy with carbon ions could surmount several central glioma resistance mechanisms by eradicating hypoxic and stem cell-like tumor cells, as well as modulating the glioma niche toward an antiangiogenic and less immunosuppressive state. Conclusively, potentially novel rationales for CIR in conquering glioma radioresistance are provided.

Authorship note: SC, ZT, and DL contributed equally to this work. WW and $A A$ are co-senior authors.

Conflict of interest: The authors declare that they have no conflict of interest.

License: Copyright 2019, American Society for Clinical Investigation.

Submitted: July 26, 2018

Accepted: December 6, 2018

Published: January 24, 2019

Reference information: JCI Insight. 2019;4(2):e123837. https://doi.org/10.1172/jci. insight.123837.

\section{Introduction}

Glioblastoma multiforme (GBM) is the most common primary brain tumor in adults. Radiotherapy has been, with surgery, the mainstay treatment of patients with GBM since the 1970s and could substantially increase the median overall survival (OS) from 3 to 4 months to approximately 12 months (1-3). Little clinical progress has been made over the past decades. The prognosis of GBM patients remains poor, with median OS of approximately 14 months after adjuvant temozolomide-based radiochemotherapy, with relevant predictive impact of inactivation of the methyl-guanine DNA methyltransferase gene promoter $(3,4)$. Local recurrence within $2 \mathrm{~cm}$ of the presurgical initial tumor margin is the main pattern of failure following treatment of $\operatorname{GBM}(1,2,5)$. Therefore, there is an urgent need for development of more effective local therapies. Emergence of heavy ion radiotherapy using carbon ions (carbon irradiation) and raster scanning technique constitutes a landmark development in the field of high-precision radiotherapy (HPR) $(1,6,7)$. HPR holds promise for escalating the dose in the tumor and improving local control while sparing normal 
tissue, e.g., in reirradiation of recurrent glioma $(7,8)$. However, previous data indicate that escalating the dose alone will most conceivably not suffice to improve outcomes in these radioresistant tumors in vivo due to a plethora of known biological mechanisms (1). A highly infiltrative growth pattern limiting proper delineation of the tumor by current imaging for HPR, existence of glioma stem cell-like cells that are proficient in repairing radiation-induced DNA damage, enhanced intratumoral networks and aberrant tumor-stroma communication with excessive angiogenesis, an immunosuppressive niche characterized by infiltration of microglia, tumor-associated macrophages (TAMs; M2 polarization), as well as recruitment of myeloidderived suppressor cells (MdSCs) were recently proposed as being among the mechanisms governing tumor recurrence $(1,5,9-13)$.

Limited in vitro studies in different glioma tumor cell lines suggest a higher cell killing effect of carbon irradiation (CIR) as compared with conventional photon irradiation (PIR) (10,14). Induction of apoptosis, autophagy, and cellular senescence are among postulated mechanisms underlying enhanced glioma cell killing by CIR (see supplemental material; available online with this article; https://doi.org/10.1172/jci. insight.123837DS1). CIR is further shown to inhibit glioma migration via decreased expression of integrins (15) and to efficiently eradicate glioma stem cell-enriched (GSC-enriched) radioresistant cultures in vitro (10). Based on these encouraging data on potential differences that may exist in radiobiology of high-linear energy transfer (high-LET) CIR versus PIR, we sought to investigate the impact of CIR on glioma-stroma communication in orthotopic, syngeneic murine xenografts as well as GSC-enriched, patient-derived xenografts.

\section{Results}

The clonogenic survival fraction (SF) is considered the gold standard for relative biological effectiveness (RBE) determination of different radiation qualities. In addition to PIR, proton irradiation (HIR) was used as a second reference of radiation quality to be compared with CIR.

Radiobiological characterization of CIR. We selected 3 of the most radioresistant patient-derived GSC cultures: NCH644, NCH441, and T325 $(10,16)$. CIR was 1.96-, 3.13-, and 1.52-fold more effective in reducing the SF by less than or equal to $10 \%$ in NCH644, NCH441, and T325 models, respectively (Supplemental Table 1 and Supplemental Figure 1; supplemental material available online with this article; https://doi.org/10.1172/jci.insight.123837DS1). This is, to our knowledge, the first report on radiobiological characterization of prototypic murine syngeneic glioma models, SMA-560/VMDk and G1261/C57B16 (Supplemental Table 1 and Supplemental Figure 2). The RBE for protons was higher than the currently used fixed 1.1 in the clinical routine, ranging from 1.16 in G1261 to 1.38 in SMA-560, with $1.154 \pm 0.134$ being the SEM \pm SD across all 5 glioma models. Next, we studied the radiosensitization effect of temozolomide (TMZ). Interestingly, the sensitizer enhancement ratio at SF 10\% for TMZ was highest when combined with CIR (1.35 in SMA-560, 1.29 in G1261, 1.23 in NCH644) compared to therapy with HIR or PIR (between 1.05 and 1.34, Supplemental Table 1 and Supplemental Figure 2). We also investigated the effect of radiation qualities on glioma migration using scratch assay and time-lapse microscopy (Supplemental Figure 3). Both PIR and CIR significantly inhibited glioma infiltration into the wound with no apparent radiation quality-specific differences.

Efficient eradication of GSC population translates to improved OS after CIR. Lack of in vivo preclinical data constitutes a major shortcoming in clinical translation of CIR. Therefore, we devised a custom platform for HPR with CIR to treat orthotopic glioma in the small animal setting (Supplemental Figure 4, A and B). Carbon ion-induced secondary positrons enable delineation of the radiation field by micro-PET imaging (Figure 1A). Consequently, the precision of CIR was confirmed by correlating the irradiated field with the Fluor ${ }^{18}$-Ethyl-Tyrosine (FET) signal that marked the metabolically active tumor region demonstrating high spatial conformity (Figure 1A and Supplemental Figure 4, A and B). NCH644 GSC-enriched xenografts were studied by fractionated irradiation with total doses of 15-Gy PIR and 6-Gy CIR (NCH644) in 3 subsequent daily fractions. The NCH441 model was treated with total dose of 7.5-Gy CIR and 25-Gy PIR in 5 subsequent daily fractions. The selection of approximately 1- to 1.5-Gy CIR versus 5-Gy PIR dose per fraction was estimated based on the in vitro RBE data. A significant inhibition of tumor growth was found in the orthotopic NCH441 model after CIR versus PIR as determined by a 20-fold decrease in bioluminescence signal between the 2 arms $(P<0.05)$ (Figure 1B and Supplemental Figure 4C). Likewise, for the NCH644 model, carbon treatment prolonged survival of mice by approximately 9 and 5 days as compared with sham-treated control $(P<0.0001)$ and PIR-treated $(P=0.02)$ mice, respectively (Figure 1C). To longitudinally quantify tumor volume, contrast-enhanced, T1-weighted MRI was used. In line with 
survival data, significant tumor growth inhibition in PIR-treated $(P<0.05)$ and CIR-treated $(P<0.0001)$ groups was achieved at median survival (12 days after irradiation) in the NCH644 model (Figure 1C). Next, the abundance of $\mathrm{CD}_{133}{ }^{+}$GSCs was examined by immunofluorescence and biodistribution studies using radionuclide $\mathrm{I}^{131}$-labeled antibody targeting the AC133 epitope of the stem cell marker CD133 ( ${ }^{131} \alpha \mathrm{CD} 133$ ) (Figure 1, D and E). Tumor uptake ratios of $\mathrm{I}^{131} \alpha-\mathrm{CD} 133$ indicated a significant relative in vivo enrichment of the radioresistant GSC population after PIR versus CIR and sham treatment $(P<0.02)$.

Tumor regression and long-term control after single-dose CIR. In dose response experiments, orthotopically implanted SMA-560 tumors in VMDk mice irradiated with single doses up to 30 Gy were investigated. Intriguingly, tumor regression with lack of recurrence after CIR at doses greater than or equal to 15 Gy were observed by longitudinal bioluminescence imaging (Figure 2A and Supplemental Figure 5A). This encouraging data led us to compare 15-Gy CIR with 15-Gy PIR or HIR, respectively (isodose comparison) in a larger prospective trial. Based on the in vitro RBE estimates, a 5-Gy CIR group was added. SMA-560 tumorbearing mice were irradiated, and tumor burden was longitudinally monitored by bioluminescence (Figure 2A and Supplemental Figure 5B). A significant delay in tumor growth was detected by bioluminescence 10 days after radiotherapy with $15-$ Gy CIR as compared with control $(P \leq 0.01), 15-G y$ PIR $(P \leq 0.003), 15-G y$ HIR $(P \leq 0.003)$, or $5-G y$ CIR mice $(P \leq 0.02)$. There was a gradual decrease of mean bioluminescence from 15-Gy PIR, 15-Gy HIR, to 5-Gy CIR. Likewise, FET PET analysis revealed a reduced tumor burden and metabolic activity after 15 -Gy CIR as compared with control $(P \leq 0.01), 15$-Gy PIR-irradiated $(P \leq 0.01)$, or 5-Gy CIR-treated $(P \leq 0.02)$ mice (Supplemental Table 2 and Supplemental Figure 7). Tumor burden further correlated with weight loss induced by tumor cachexia (Supplemental Figure 5B). Consequently, efficient tumor control led to an increase of animal weight in the 15-Gy CIR group.

Fractionated CIR prolonged survival in a syngeneic model. Based on in vitro clonogenic survival data, 3.25Gy CIR was depicted to be correlated with 5-Gy PIR for orthotopic G1261 in a syngeneic C57B16 mouse model. However, CIR treatment significantly prolonged survival of G1261 tumor-bearing mice by approximately 10 and 7 days as compared with sham-treated control $(P<0.001)$ and PIR-treated $(P<0.05)$ mice, respectively (Figure 2C). Accordingly, a lower tumor FET uptake, reflecting decreased gross tumor burden and metabolic activity, was found in CIR-treated mice compared with PIR-treated mice $(P \leq 0.05)$ and sham-treated control mice ( $P \leq 0.01)$ (Supplemental Table 2). Contrast-enhanced MRI was performed and tumor volumes were quantified in all 3 groups 12 days after therapy. CIR mice displayed significant delay in tumor growth as compared with PIR $(P=0.03)$ or control $(P<0.001)$ mice. With an average volume of $30 \mu 1$, CIR-treated tumors were 2.1- or 2.7-fold smaller as compared with PIR-treated mice or sham-treated control mice, respectively (Figure 2C). Despite a comprehensive existing preclinical and clinical CIR neurotoxicity profile, we evaluated non-tumor-bearing mice treated with the same schemes by serial MRI and observed no CIR-mediated toxicity up to 360 days after irradiation (Supplemental Figure 6).

Reduced tumor perfusion and angiogenesis after CIR. Tumor MVD was reduced in SMA-560 tumor-bearing mice after 5-Gy $(P=0.03)$ and 15-Gy $(P \leq 0.01) \mathrm{CIR}$ as compared with sham-treated control mice (Figure 2B). Moreover, MVD was significantly reduced after $15-$ Gy CIR as compared with $15-$ Gy PIR $(P=0.03)$ and 5-Gy CIR $(P \leq 0.01)$. Similarly, tumor perfusion assessed by $\mathrm{G}^{68}$-RGD peptide micro-PET was reduced after irradiation with 15 -Gy CIR as compared with $15-$ Gy PIR $(P \leq 0.02)$ or 5 -Gy CIR $(P \leq 0.05)$ treatment arms (Supplemental Table 2). Likewise, the MVD of G1261 and NCH644 tumors was significantly reduced after fractionated CIR as compared with control $(P<0.0001)$ (Figure $2 \mathrm{C}$ and Supplemental Figure 8A). CIR treatment was more effective in reducing MVD as compared with PIR in the G1261 model $(P<0.001)$ (Figure 2C). These data suggest potent antiangiogenic effects of CIR in the glioma microenvironment, most notably in syngeneic models.

Differential transcriptome response to CIR versus PIR. To identify the potential differences in tumor response as a function of radiation quality, genome-wide transcriptional analysis was performed on in vivo tumor material of the 2 syngeneic models. First, The Cancer Genome Atlas's classification, based on 840 gene expression signatures, of glioblastoma into transcriptional subtypes was applied to data using classification to nearest centroids (ClaNC) minimum distance to class centroids. This analysis resulted in a relatively robust assignment of the G1261 tumors to the "classical" subtype. PIR led to a uniform switch of the G1261 subtype toward a "neural" phenotype. In contrast, GL261 tumors treated with CIR remained mainly classified in the prognostically more favorable "classical subtype" (Figure 3 and Supplemental Figure 9).

Next, from the differentially expressed genes as a function of radiation quality in SMA-560 tumors, 595 transcripts with inverse regulation pattern after PIR versus CIR were selected using stringent criteria 
A
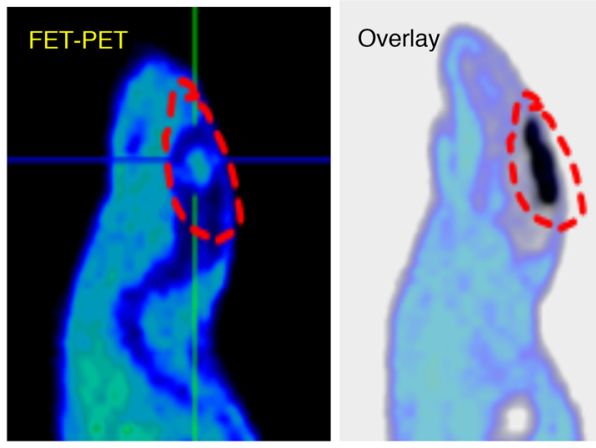

B

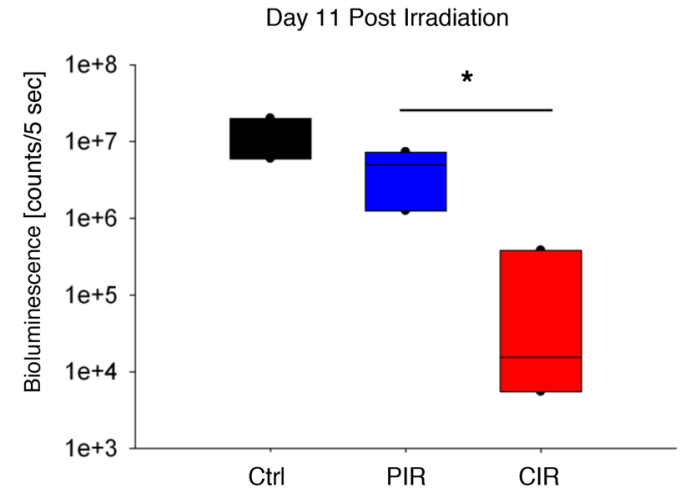

D
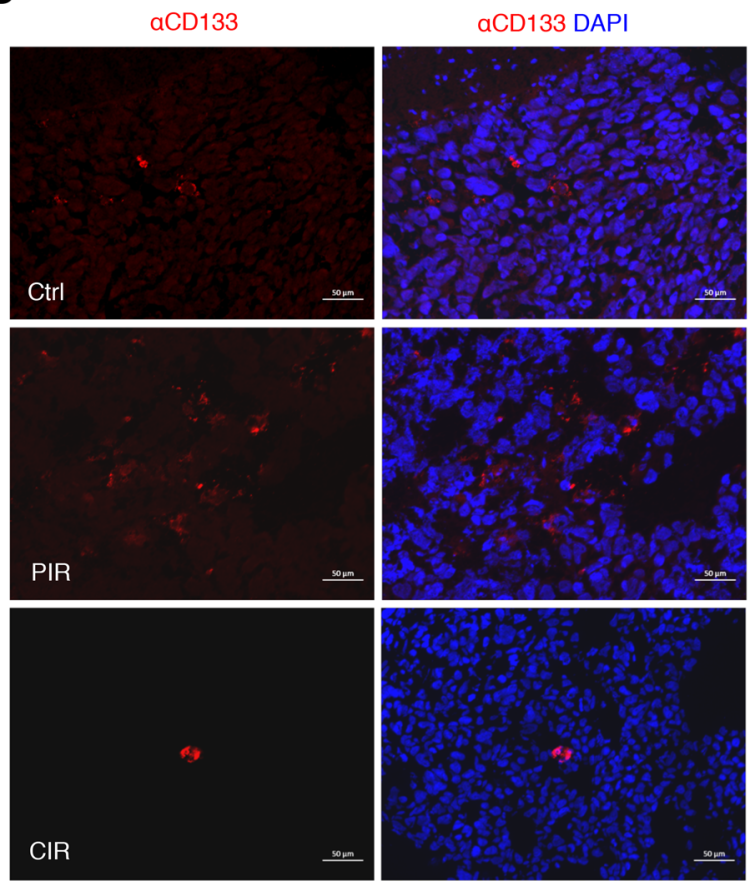

C
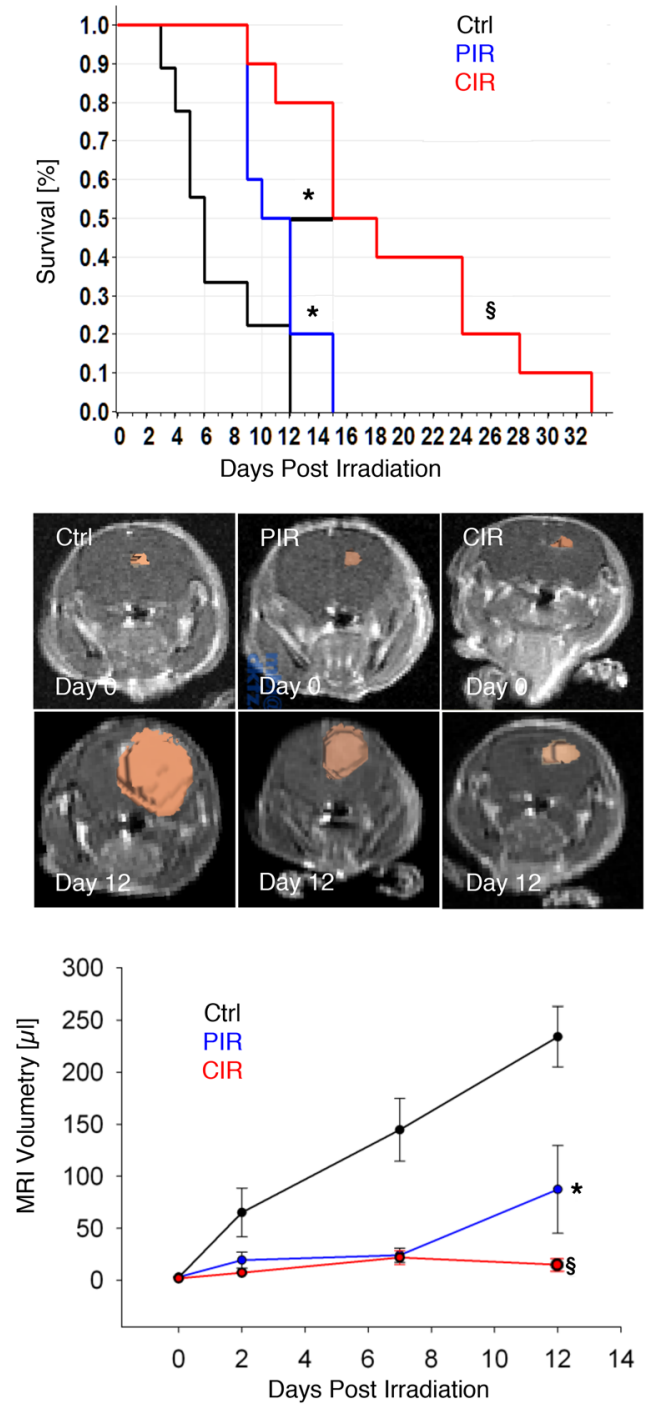

E

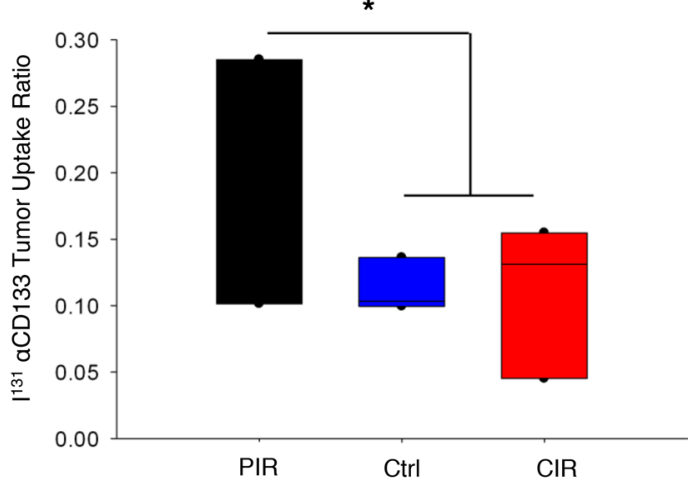

Figure 1. Effect of CIR on patient-derived, CSC-enriched xenograft models. Establishment of small animal CIR in orthotopic glioma models. (A) Verification of administered dose by overlay of CIR-induced secondary positrons ( ${ }^{11}$ micro-PET, black) and tumor uptake of ${ }^{18} \mathrm{~F}$-fluoro-ethyl-tyrosine (FET) PET, circled in red at the intersection of the horizontal and vertical lines, indicating precision treatment. (B) Significant inhibition of tumor growth after CIR ( $5 \times 1.5$ Gy) versus PIR ( $5 \times 5$ Gy) and sham-treated control in an orthotopic xenograft model derived from patient NCH441 GSCs $(n=3)$. Tumor growth was monitored longitudinally by bioluminescence (see also supplemental material). Ctrl: control. (C) CIR (6 Gy, $n=10)$ significantly prolonged animal survival compared with PIR (15 Gy, $n=10$ ), both administered in 3 consecutive daily fractions in the NCH644 GSC model. Kaplan-Meier survival estimates and tumor growth kinetic via MRI-based tumor volumetry are shown. (D and E) PIR $(n=5)$ led to a relative enrichment of the GSC population compared with control $(n=3)$ and CIR $(n=5)$ as determined by NCH644 tumor ${ }^{131} \alpha-C D 133$ uptake ratio and 
biodistribution studies (supplemental material). The lack of selection for $\alpha$-CD133+ GSCs (red) is also elucidated by representative immunofluorescence staining. Nuclei counterstained using DAPI (blue). Box and whisker plots represent median, interquartile, minimum, and maximum of all data points. ${ }^{s} P<0.0001,{ }^{*} P<0.05$ versus control, when shown over a box, or versus the indicated irradiation treatment. Scale bar: $50 \mu \mathrm{m}$.

(ANOVA, $P<0.01$ after Bonferroni's multiple testing correction). Among these, 265 were upregulated after CIR while downregulated after PIR and were enriched in GeneGo process maps and functional processes associated with cellular differentiation (neurogenesis and adhesion), response to oxidative stress, multiple metabolic processes (including oxidative phosphorylation and lipid metabolism), redox balance (glutathione), as well as calcium transport (Figure 3, Supplemental Figure 10, Supplemental Lists 1, 2, 5, and 6). In contrast, 323 transcripts were downregulated by CIR while upregulated by PIR relative to sham-treated control (Figure 3 and Supplemental Figure 10, Supplemental Lists 3-6). In line with MVD and perfusion studies, these genes were enriched for GeneGo process maps associated with angiogenesis, notch signaling, epithelial-mesenchymal transition (EMT), and remodeling of ECM. Most strikingly, functional processes and pathways related to angiogenesis and hypoxia-induced signaling (HIF1A, endothelin, PEDF); recruitment of microglia; TAMs and bone marrow-derived myeloid cells (SDF1/CXCR4 signaling); inflammatory and immune processes (IL6-JAK-STAT/NOTCH1-NFKB signaling); and EMT (PDGF/EGFR/TGFb-WNT signaling) were enriched for CIR-downregulated genes.

CIR promotes an immunopermissive tumor niche. Based on transcriptome data and gene regulatory network analysis, the composition of tumor stroma and differential regulation of signaling cascades was further evaluated. PIR potently induced SDF1 expression correlating with high tumor influx of CD11 $\mathrm{b}^{+}$microglia/ TAMs and $\mathrm{CD} 11 \mathrm{~b}^{+} \mathrm{GR} 1^{+}$immunosuppressive MdSCs in all 3 investigated tumors (Figure 4A and Supple-

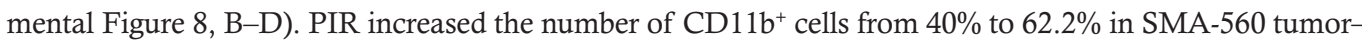
bearing mice, from $35 \%$ to $55 \%$ in G1261 tumor-bearing mice ( $P=0.03$ ), and from $2.5 \%$ to approximately $4.5 \%(P=0.03)$ in NCH644 tumor-bearing mice as compared with the control group. In contrast, CD11 ${ }^{+}$ cell populations were significantly reduced after CIR in all models in comparison with control $(P<0.05)$ and PIR $(P<0.0001)$ groups (Figure 4A and Supplemental Figure 8B). Irradiation with 5-Gy CIR also significantly reduced tumor $\mathrm{CD} 11 \mathrm{~b}^{+}$cell number as compared with the $15-\mathrm{Gy}$ PIR group $(P \leq 0.01)$ in the SMA560 model (Figure 4A). Moreover, the fraction of $\mathrm{Gr}^{+} \mathrm{MdSCs}$ was reduced after 15-Gy CIR as compared with control $(P<0.001)$, PIR $(P \leq 0.01)$, HIR $(P<0.001)$, and $5-G y$ CIR in the SMA-560 tumors $(P \leq 0.02)$ (Figure $4 \mathrm{~A}) . \mathrm{Gr}^{+}$cell populations were also reduced by fractionated CIR in G1261 and NCH644 models (Figure 4A and Supplemental Figure 8C). Regulation of SDF1 as a key microglia and myeloid cell attractant was further investigated. Enhanced SDF1 expression levels in tumors after PIR versus downregulation after CIR was found (Figure 4A and Supplemental Figure 8D). SDF1 was downregulated only by CIR and not PIR in the SMA-560 model (Figure 4A). SDF1 expression was significantly reduced in tumors treated with CIR as compared with control $(P<0.01)$ in all 3 tumor models.

Virtual microdissection of G1261 and SMA-560 tumors using the CIBERSORT algorithm revealed a significant polarization of the microenvironment toward an M2 immune phenotype by PIR, whereas the population of M1 TAMs was significantly reduced by PIR (in the SMA-560 model) compared with the sham-treated control $(P=0.002)$ (Figure 4B and Supplemental Figure 11). In line with other immune cell infiltrate parameters, an inverse regulation pattern was found for both M1 and M2 populations between CIR versus PIR treatment in both murine models. This was accompanied by a lower abundance of cytotoxic CD8 ${ }^{+} \mathrm{T}$ cells after PIR and markedly increased presence of $\mathrm{CD} 8^{+}$cells in CIR-treated tumors (Figure 4B and Supplemental Figure 11).

CIR eradicates hypoxic tumor cells and inhibits the SDF1/CXCR4-dependent microglia influx. Next, we aimed to functionally validate the impact of radiation quality-dependent regulation of SDF1/CXCR4 signaling in recruitment of microglia using an in vitro coculture migration assay. Exposure of SMA-560 tumor cells in the lower chamber of a transwell system increased microglia migration (Figure 5A). Selective PIR of tumor cells in the lower chamber further enhanced the migration of microglia by approximately 2.5 -fold $(P<0.03)$. Coculture- and PIR-induced microglia recruitment was reversible by the addition of CXCR4 inhibitor AMD3100. In contrast, CIR significantly inhibited tumor-induced recruitment of microglia (Figure 5A). These data suggest a pivotal role for the SDF1/CXCR4 axis in radiation-induced recruitment of microglia into tumor stroma, which is abrogated by CIR. Upregulation of HIF1- $\alpha$ in PIR-resistant, hypoxic glioma cells was shown to promote SDF1 expression (17). In line with pathway enrichment analysis of transcriptome data, HIF1- $\alpha$ expression paralleled SDF1 expression as confirmed by real-time 
A
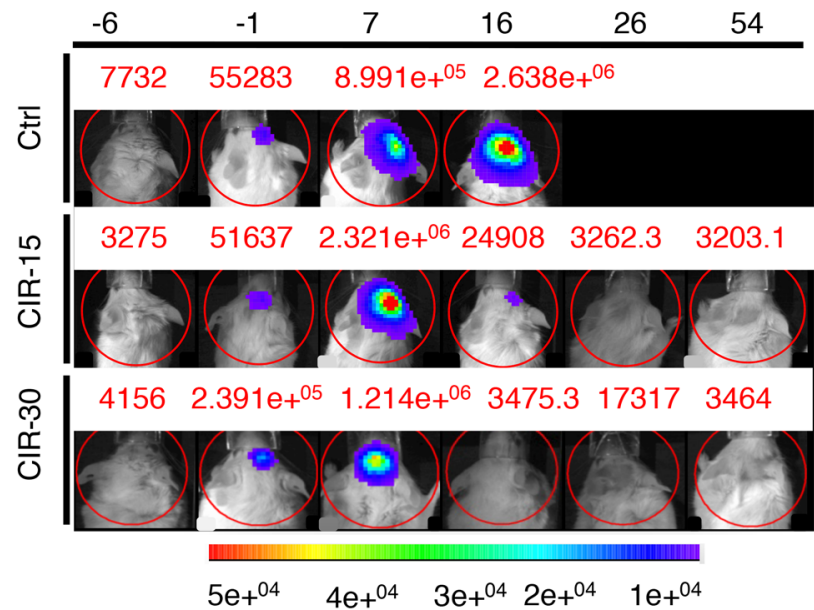

B
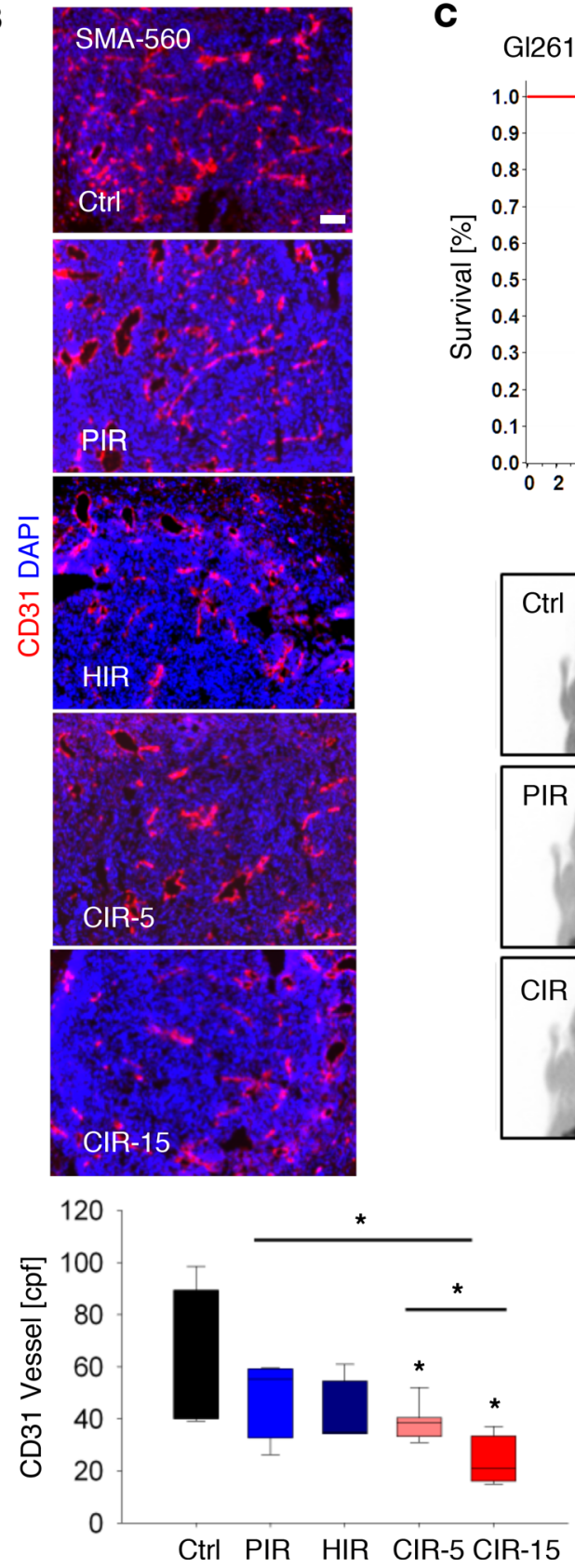

C

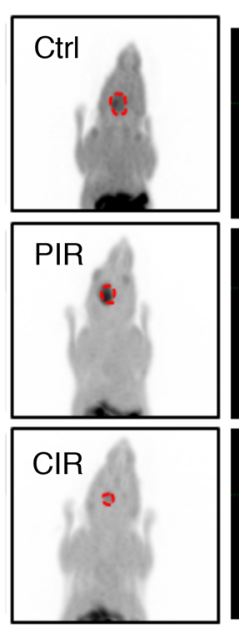

SMA-560

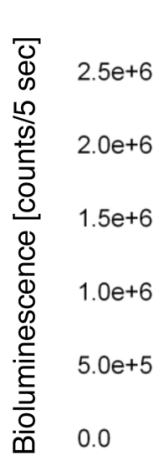

Day 10 Post Irradiation
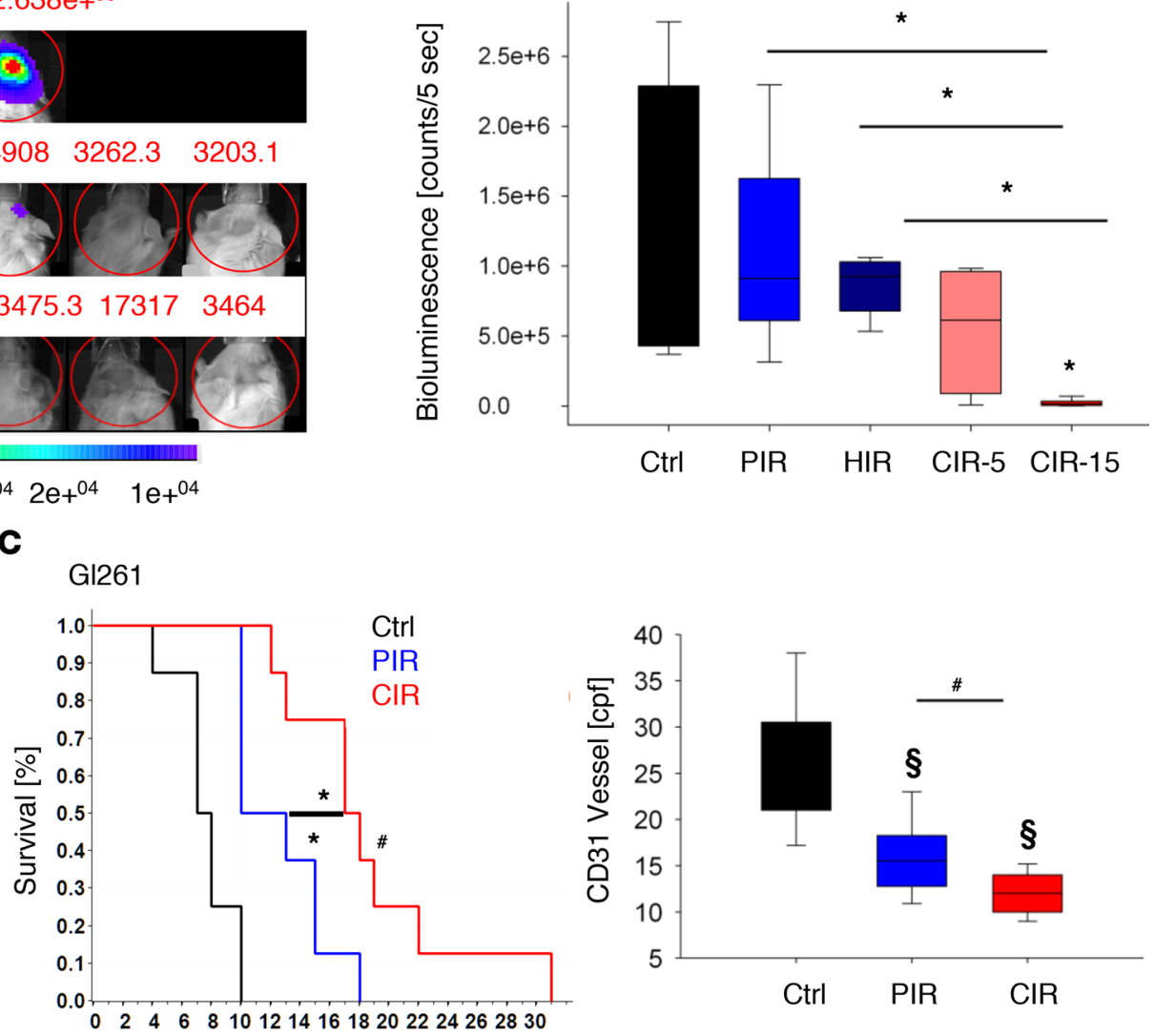

Days Post Irradiation
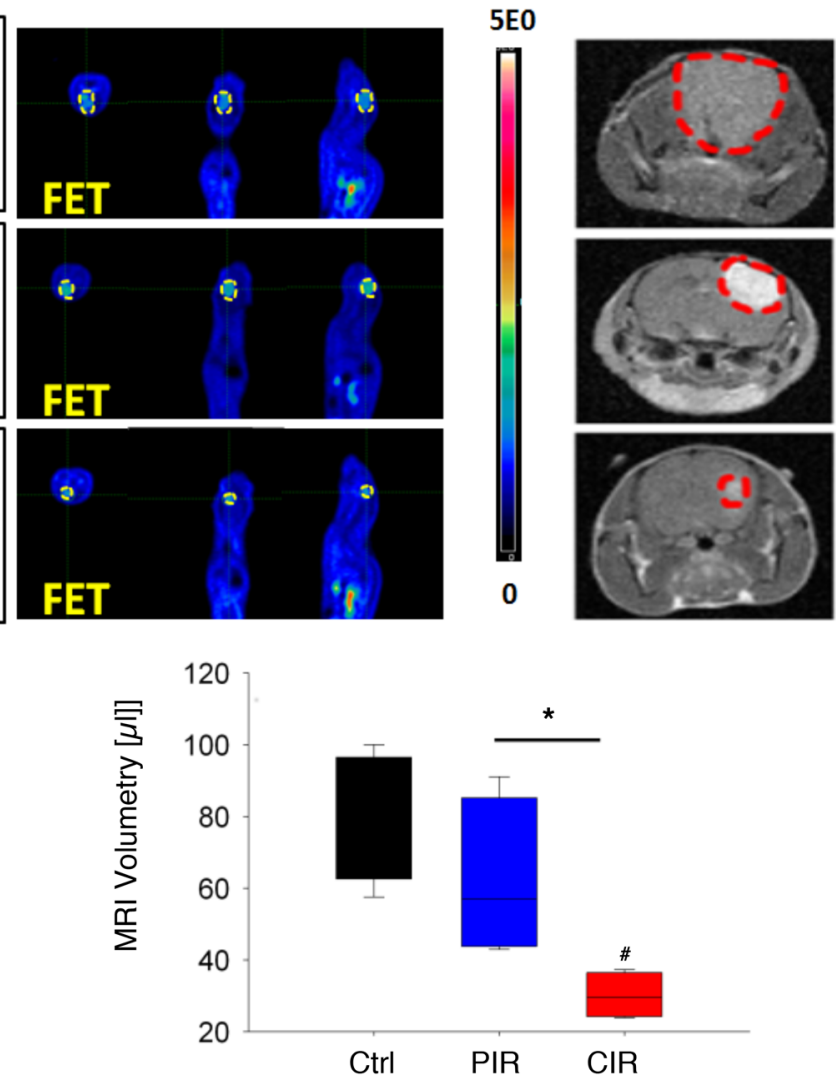
Figure 2. Beneficial effect of CIR in syngeneic, orthotopic murine models. (A) Comparison of PIR, HIR, and CIR in an intracranial syngeneic SMA-560 murine glioma model. Tumor control at single doses $\geq 15$-Gy CIR was observed. Tumor growth ( $n=2 /$ study arm) was longitudinally monitored by bioluminescence at $-6,-1,7,16,26$, and 54 days after irradiation. Next, SMA-560 tumors were irradiated with 15-Gy PIR ( $n=12), 15-\mathrm{Cy}$ HIR ( $n=12), 15-\mathrm{Cy}$ CIR (CIR-15, $n=$ 12), or 5 -Gy CIR (CIR-5, $n=12$ ). Tumor growth inhibition was significant only for the CIR-15 group versus all other arms at day 10 after irradiation as determined by bioluminescence. Representative photomicrographs of tumor vascular staining by anti-endothelial CD31 marker (red) are shown. (B) Nuclei counterstained using DAPI (blue). cpf, counts per field. (C) Likewise, in the GI261 glioma model, irradiation with 6-Gy CIR significantly prolonged survival (control $n$ $=8$, PIR $n=8$, CIR $n=8)$ compared with 15-Gy PIR, both administered in 3 consecutive daily fractions. This effect also correlated with reduced microvascular density (MVD) ( $n=6 /$ study arm), metabolism by micro-FET PET imaging (tumor margins delineated in yellow), and tumor size measured by volumetry using T1-weighted contrast-enhanced (T1CE) MRI $(n=4)$ (hyperintense gross tumor area is contoured by red dashed lines). Bioluminescence, MRI tumor volumes, and CD31+ blood vessels per field data are represented as box-and-whisker plots showing median, interquartile, the minimum and maximum of all data points. ${ }^{s} P<0.0001,{ }^{\#} P<0.001,{ }^{*} P<0.05$ versus control, when shown over a box, or versus the indicated irradiation treatment. Scale bar: $50 \mu \mathrm{m}$.

quantitative reverse transcription PCR (Figure 5B). HIF1- $\alpha$ expression was significantly increased after PIR whereas downregulated after CIR in SMA-560 tumors $(P<0.05)$. The presence of oxygen is important for generation of ROS after PIR. Hence, a higher dose is required to achieve the same SF under hypoxic conditions, a phenomenon that is described with the oxygen enhancement ratio (OER). In contrast, highLET CIR is postulated to be less dependent on oxygen concentration. These hypotheses were validated for both syngeneic models (Figure 5C and Supplemental Figure 12). While OER was increased up to 1.63 after PIR in SMA-560 tumor cells, no significant difference was observed under normoxic versus hypoxic conditions after CIR (OER 1). Therefore, hypoxia rendered tumor cells resistant to PIR but did not alter their sensitivity to CIR.

CIR versus PIR treatment of patients with recurrent glioma. Two patients, diagnosed with astrocytoma (WHO grade II), were treated with 54-Gy PIR in 27 fractions. Tumor recurrence as high-grade glioma (HGG) was recorded after 9 and 11 years, respectively. One patient was reirradiated with 36-Gy PIR in 18 fractions while the other patient was treated in a pilot trial (8) with 36-Gy-equivalent CIR in 11 fractions (Figure 5, D and E). Progression-free survival was 5.2 and 11.3 months for the PIR- and CIR-treated patient, respectively. MRI sequences for T1CE (area of disruption of the blood-brain barrier), T2-weighted FLAIR (representing edema), ADC (correlating with cellularity), and perfusion-weighted MRI (indicating tumor vascularity) were selected for analysis. After PIR, a gradual increase of FLAIR- and ADC-defined volumes was found over the follow-up period despite decreased contrast enhancement on T1CE sequences. Conversely, the CIR-treated tumor showed a clear decrease in volume (T1CE). This was in concordance with information obtained from T2 FLAIR and ADC sequences. Perfusion sequences showed a decrease in cerebral blood flow for the tumor volume within 1.5 months. Both patients showed a high agreement between the T2 FLAIR-defined and ADC-defined volumes. Despite these encouraging data in $n=1$ representative patient for which serial imaging and long-term follow-up of the primary tumor, conversion to recurrent HGG and post-photon/carbon ion therapy were available, only prospective trials could confirm the value of our preclinical observations. Indeed, preliminary data on the first collectives of recurrent HGG patients reirradiated with raster scanning carbon ions are promising, indicating that progress mainly occurred outside the high-dose field, in contrast with the predominantly central progression pattern found after PIR $(18,19)$.

\section{Discussion}

This study reports on beneficial effects of particle therapy with heavy ions by using CIR to overcome central radioresistance mechanisms governing local failure and poor prognosis of HGG (Figure 6). HGGs were among the first solid tumors for which an intratumoral hierarchy with radioresistant tumor stem cells in the apex was postulated (9). In the present study, CIR efficiently eradicated several radioresistant GSC cultures in vitro, which is in line with recent findings (10). Biodistribution studies revealed an enrichment of the $\mathrm{CD} 133^{+}$GSC population in vivo after conventional radiotherapy with PIR but not CIR. To our knowledge, this is the first report suggesting the high efficacy of this novel therapy to eradicate tumor cells independent of their hierarchical status in vivo in orthotopic, patient-derived xenograft models. Indeed, the ability of high-LET carbon ions to cause complex, unrepairable DNA damage via direct interaction with the genome (20) was postulated to reduce its dependency on the presence of oxygen $(6,21)$. Consequently, it was hypothesized that carbon ions may overcome classical radioresistance (22) that is attributed to tumor stem cell proficiency to quench ROS and repair DNA double-strand breakages induced by low-LET ionizing radiation (9). Our data clearly support this hypothesis. Moreover, our data indicate enhanced efficacy of CIR in vivo could be in part attributed to its positive effects at the tumor-stroma interface. 

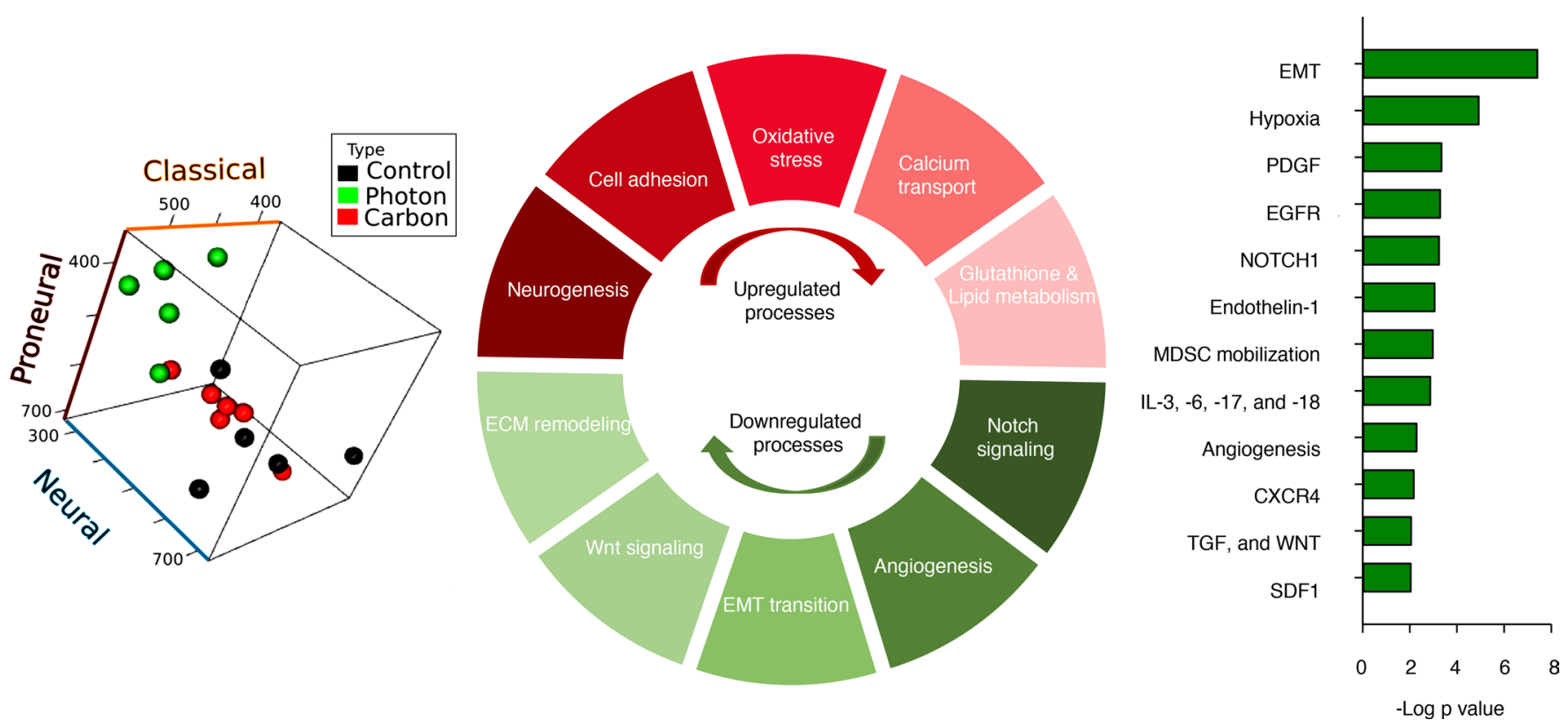

Figure 3. Transcriptomic switches associated with carbon ion irradiation in glioma models. Transcriptome analysis of in vivo Gl261 tumor samples ( $n=5$ control, photon, $n=6$ carbon) revealed that sham-treated control tumors represent predominantly a "classical" gene expression subtype. Conventional radiotherapy (photon) led to a uniform switch of this gene expression subtype toward a "neural" phenotype. In contrast, Gl261 tumors treated with carbon ions (carbon) mainly retained their gene expression subtype and were classified in the prognostically more favorable "classical" subtype. Graphical depiction of subtype changes in the proneural-neural-classical space for PIR versus CIR is shown (see also supplemental material). In the SMA-560 model ( $n=4 /$ study arm), 595 differentially expressed transcripts as a function of radiation quality were identified $(P<0.01$ after Bonferroni's multiple testing correction). Significantly enriched pathways identified among the 265 upregulated (red), and 323 downregulated (green) transcripts after CIR are presented. Bars represent negative log $P$ values, representing the probability for enrichment based on gene ontology mapping to arise by chance. Of note, numerous gene ontologies related to tumor microenvironment (hypoxia, angiogenesis), growth factors, and cytokines, as well as modulators of an immunosuppressive niche (SDF1/CXCR4, TGF, MdSC mobilization), were found to contain genes with inverse regulation pattern, i.e., decreased expression after carbon ion exposure whereas upregulation by conventional PIR. SDF1: stromal cell-derived factor 1.

Lack of preclinical models limited detailed analysis of glioma-stroma communication in response to particles. Here, we successfully developed small-animal particle irradiation in orthotopic mouse models. To study tumor crosstalk with the murine microenvironment under faithful constraints, 2 syngeneic orthotopic models were used. Our data equivocally demonstrate the efficacy of CIR to enhance glioma immunity by reducing the abundance of $\mathrm{CD} 11 \mathrm{~b}^{+}$microglia, $\mathrm{M} 2$ polarization, and the influx of immunosuppressive MdSCs, correlating with enhanced infiltration of $\mathrm{CD}^{+} \mathrm{T}$ cells and decreased activation of the SDF1/ CXCR4 axis. Although both radiotherapy options reduced tumor migration, were radiosensitized by TMZ, and elicited antiangiogenic effects, the latter 2 results were more pronounced after CIR. Importantly, CIR efficiently eradicated residual radioresistant, hypoxic tumor cells, correlating with the observed switch of transcriptome signatures related to hypoxia-associated pathways. In line with efficient ablation of hypoxic cells, reduced HIF1- $\alpha$ expression as a central upstream regulator of the SDF1/CXCR4 axis was found in CIR-treated tumors in vivo. Conclusively, carbon ions could overcome multiple central mechanisms of classical radioresistance in tumor cells and in the tumor niche interface.

Although high influx of $\mathrm{CD}_{1} 1 \mathrm{~b}^{+}$and bone marrow-derived myeloid cells as well as $\mathrm{M} 2$ polarization are well characterized as negative prognosticators in glioma and mechanistically linked to therapy refractoriness, escape from tumor dormancy, and local recurrence $(12,23,24)$, therapeutic strategies to reverse these phenotypes are still urgently awaited. Antibodies against $\mathrm{CD} 11 \mathrm{~b}^{+}$or FDA-approved inhibitors of CXCR4, such as the AMD3100 used in our study, were postulated to prevent glioma radioresistance and local relapse in experimental models (25) but are still awaiting clinical validation. It was hypothesized that $\mathrm{CD} 11 \mathrm{~b}^{+} \mathrm{Tie}^{+}$bone marrow-derived cells contribute via differentiation to formation of novel vessels and tumor relapse after glioma irradiation (25). This glioma vasculogenesis principle would distinguish brain tumors from most solid tumors, where angiogenesis, formation of new vessels from preexisting vasculature, is the prevailing principle supporting tumor growth (5). In our study, we failed to identify CD11b+ $\mathrm{b}^{+} 2^{+}$cells in all utilized tumor models (data not shown). Another interesting phenomenon observed in our study was the switch of the gene expression subtype 
A

CD11b DAPI
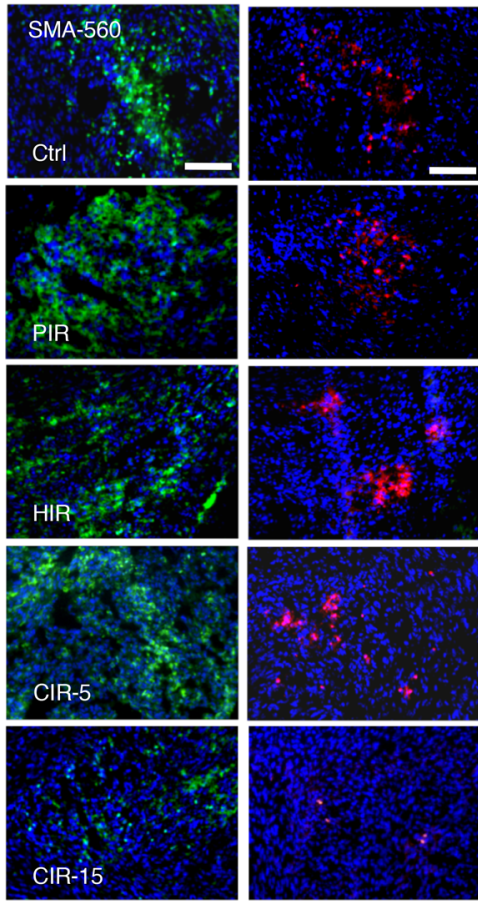

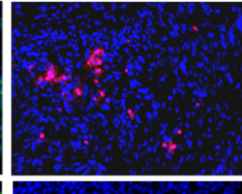

Gr1 DAPI
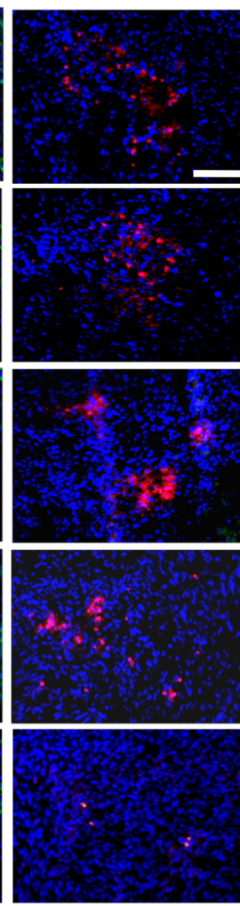

SDF1 DAPI
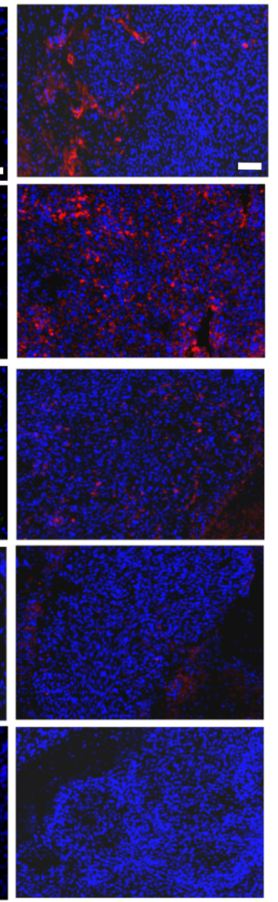
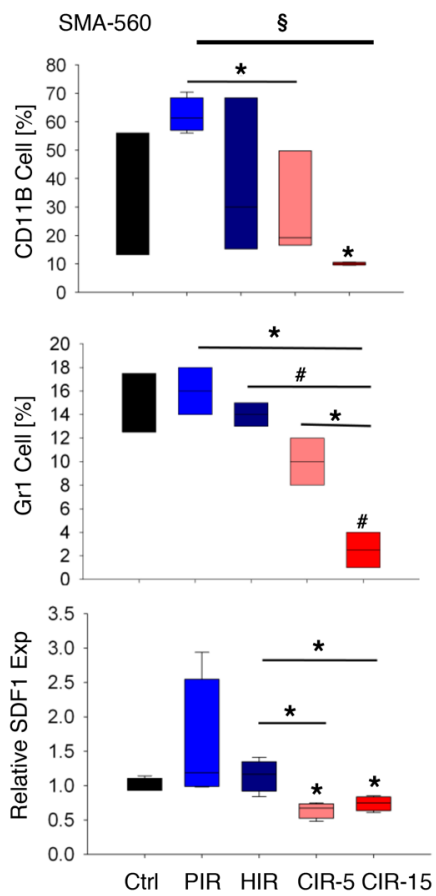
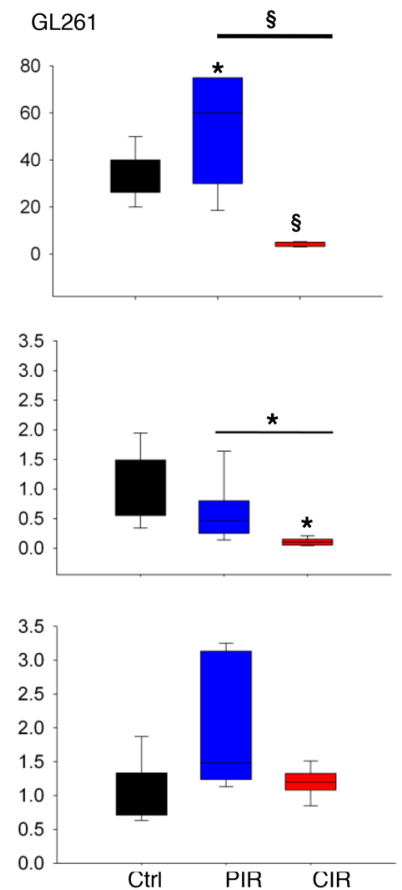

\section{B}
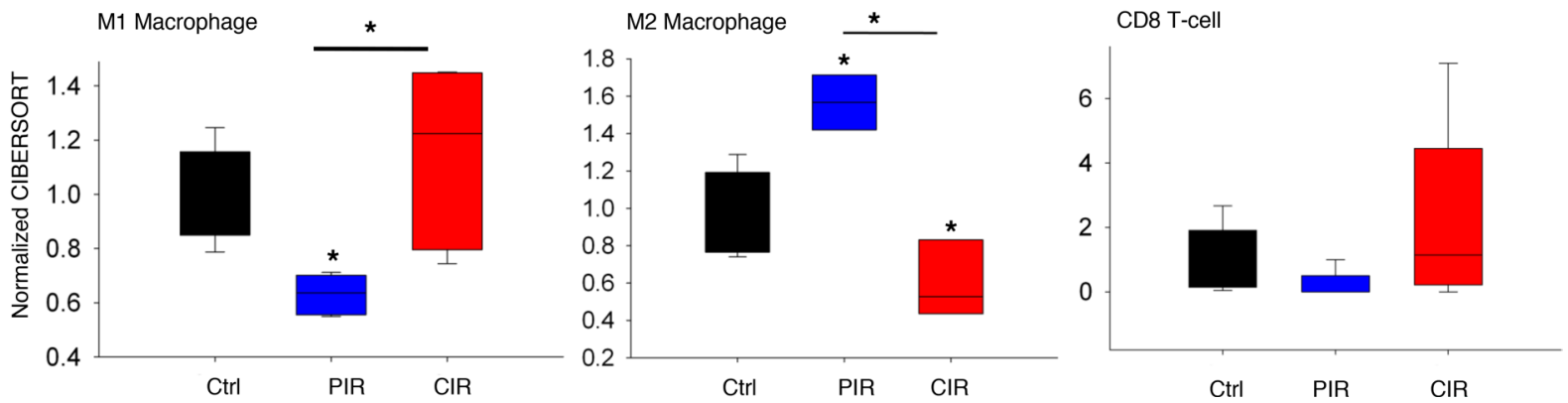

Figure 4. CIR generates an immunopermissive glioma niche. (A) In line with these observations, reduced influx of CD11b+ microglia and myeloid cells, including $\mathrm{Gr}^{+}$MdSCs, after CIR was confirmed and correlated with decreased SDF1 levels (SMA-560 $n=3-4$, GI261 $n=6$ ). Exp: expression. (B) In silico cell sorting identified a switch in macrophages/microglia polarization toward an M2 phenotype after PIR, which was accompanied by reduced abundance of CD8 ${ }^{+}$ T cells (control $n=5$, PIR $n=3-5$, CIR $n=3-5$ ). However, a reverse trend was found after CIR, indicating lack of immunosuppressive signal and accelerated glioma immunity. Box and whisker plots represent median, interquartile, minimum, and maximum of all data points. ${ }^{s} P<0.0001,{ }^{\sharp} P<0.001,{ }^{*} P<0.05$ versus control, when shown over a box, or versus the indicated irradiation treatment. Scale bar: $50 \mu \mathrm{m}$ (CD11b, Gr1).

after conventional radiotherapy. The lack of this switch after CIR indicates that alterations within the tumor microenvironment may interfere with glioma subtype assignment. In line with our observation, tumor evolution of glioma-intrinsic gene expression subtypes was recently reported to associate with immunological changes in the microenvironment (23). Implementation of more advanced single-cell transcriptome analysis (26) and image-guided isolation of cells may allow deconvolution of current bulk tumor gene signature results. For example, in addition to the hypoxia/SDF1/CXCR4 axis, interconnectedness of other processes may be deciphered, such as the recent link between GSCs and MdSCs and formation of an immunosuppressive glioma niche (27).

Together, compensatory mechanisms of photon radiotherapy, e.g., upregulation of angiogenic, stromamodulating, and other tumor-evasive signals, contribute to tumor radioresistance and have emerged as novel, promising therapeutic targets to enhance radiotherapy effects $(1,5)$. Lack of induction of these compensatory and evasive mechanisms by carbon ions opens a new venue for development of novel therapy strategies for this devastating disease. However, clinical experiences (19) reported here show that despite these advantages of CIR, tumors progress and ultimately relapse after monotherapy. It is conceivable that combination therapies will be needed to translate the beneficial effects of carbon ions into an enduring 
A

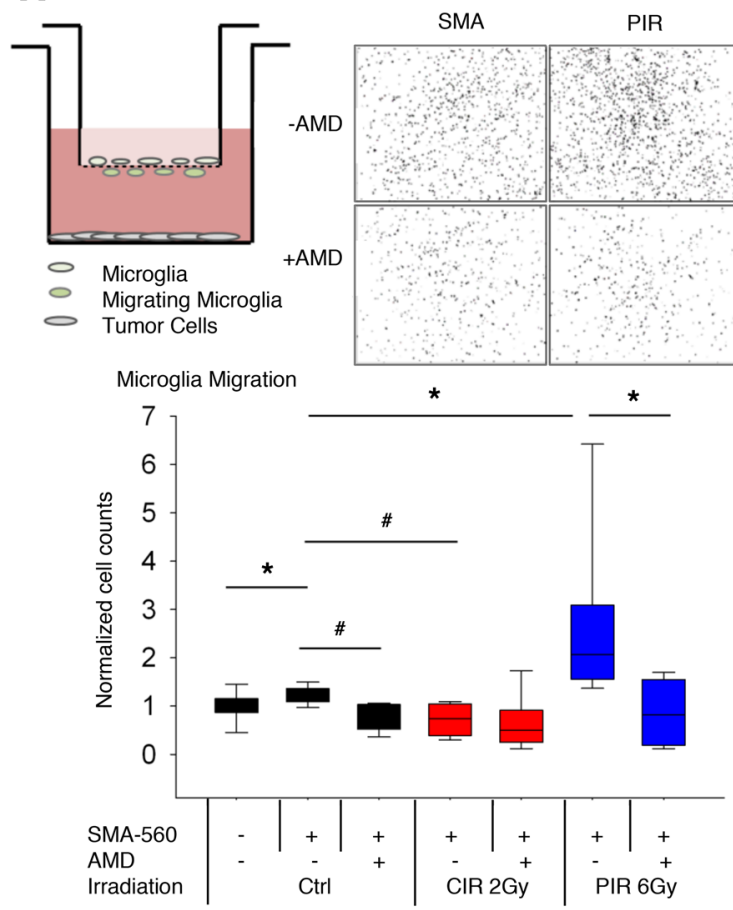

D
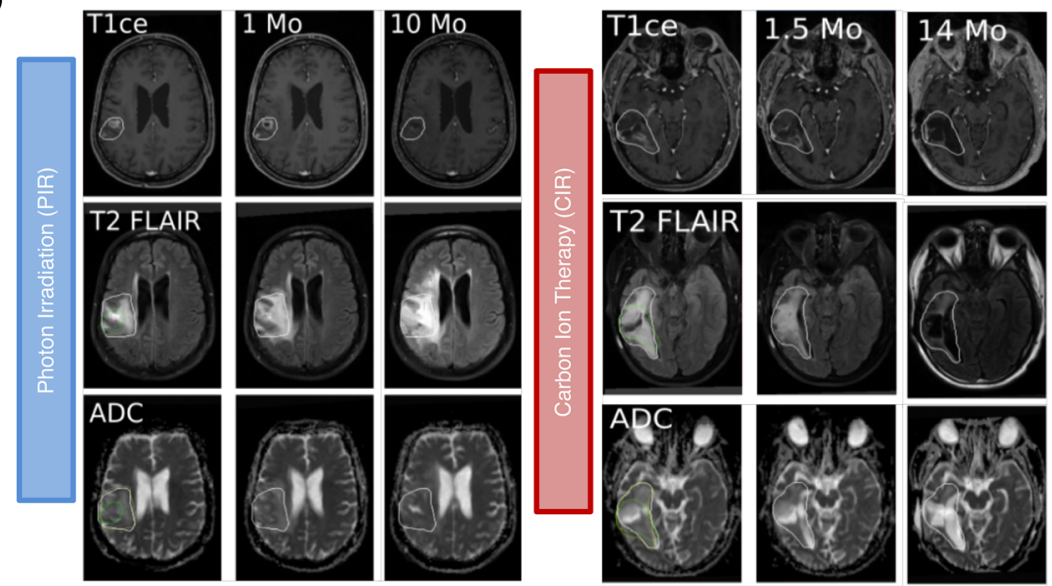

E

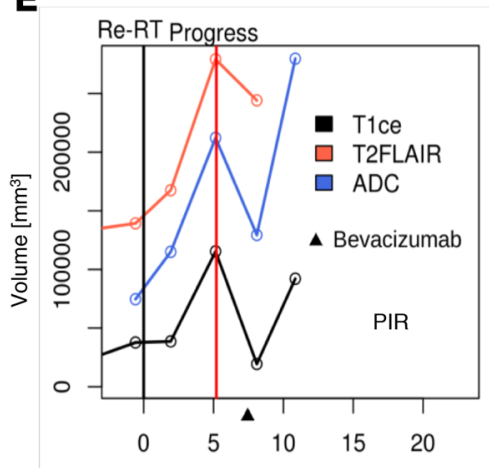

Time [months]

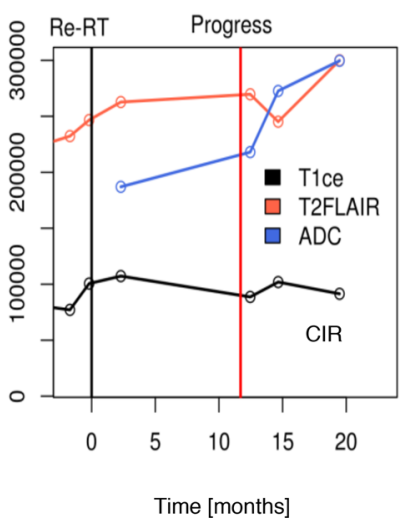

Time [months]
B
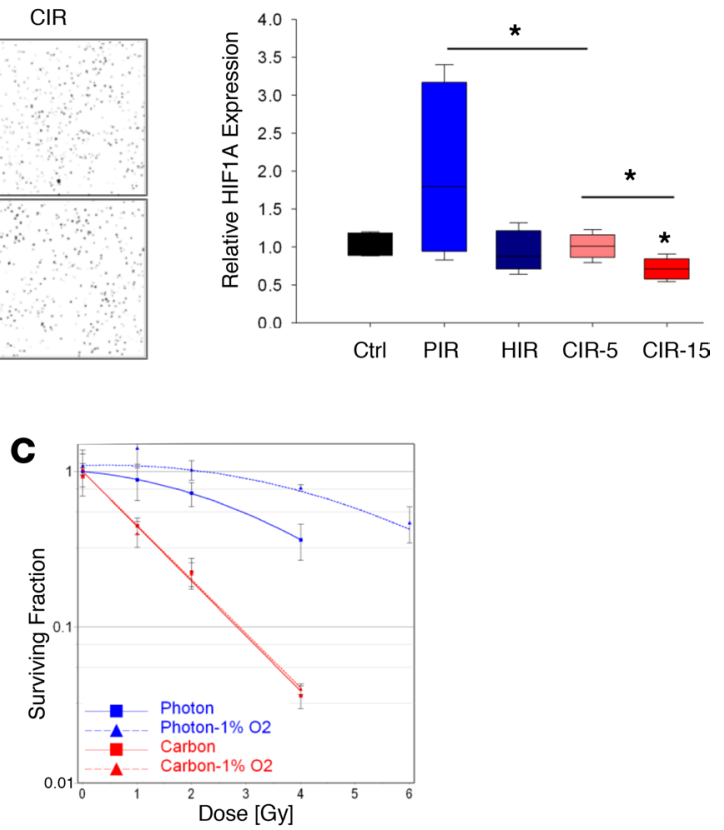

Figure 5. Functional validation of CIR effects on tumor hypoxia, HIF1- $\alpha$ /SDF1/CXCR4 axis, and tumor recruitment of microglia. (A) Irradiation of tumor cells (bottom) enhanced tumor-induced migration of microglia (embryonic stem cell-derived microglia, in transwell insert). This effect was functionally reversed by inhibition of the SDF1/CXCR4 pathway (AMD3100). In contrast, reduced influx of microglia was found after irradiation of tumor cells with carbon ions. Representative photomicrographs of infiltrated microglia nuclei on the bottom side of the transwell in coculture with SMA-560 cells in the lower chamber are shown. (B) Enhanced HIF1- $\alpha$ expression in vivo in SMA-560 tumors treated with PIR versus reduced expression after CIR suggest efficient ablation of hypoxic tumor cells by CIR. Box and whisker plots represent median, interquartile, minimum, and maximum of all data points. Indeed, clonogenic survival assay clearly demonstrated overlapping tumor sensitivity to carbon ions independent of the oxygen concentration. (C) In contrast, tumors under mild hypoxia ( $1 \% \mathrm{O}_{2}$, dotted lines) demonstrated relative radioresistance to conventional PIR. Bars indicate OER for photon and carbon ions. (D) Effects of CIR versus PIR in reirradiating patients with HGG. Alteration of tumor pathophysiology determined over time (months, Mo) correlated with improved time to progression in CIR-treated patients. Green: T1CE-defined tumor volume; yellow: T2 fluid-attenuated inversion recoverydefined (FLAIR-defined) tumor volume, apparent diffusion coefficient (ADC). (E) Longitudinal follow-up of different MRI derived tumor volumes. Time point of reirradiation (Re-RT, black vertical line) and detection of tumor progress (Progress, red vertical line), as well as treatment with antiangiogenic agent Bevacizuman (an antibody against VEGF). ${ }^{\#} P<0.001,{ }^{*} P<0.05$ versus control, when shown over a box, or versus the indicated irradiation treatment. 

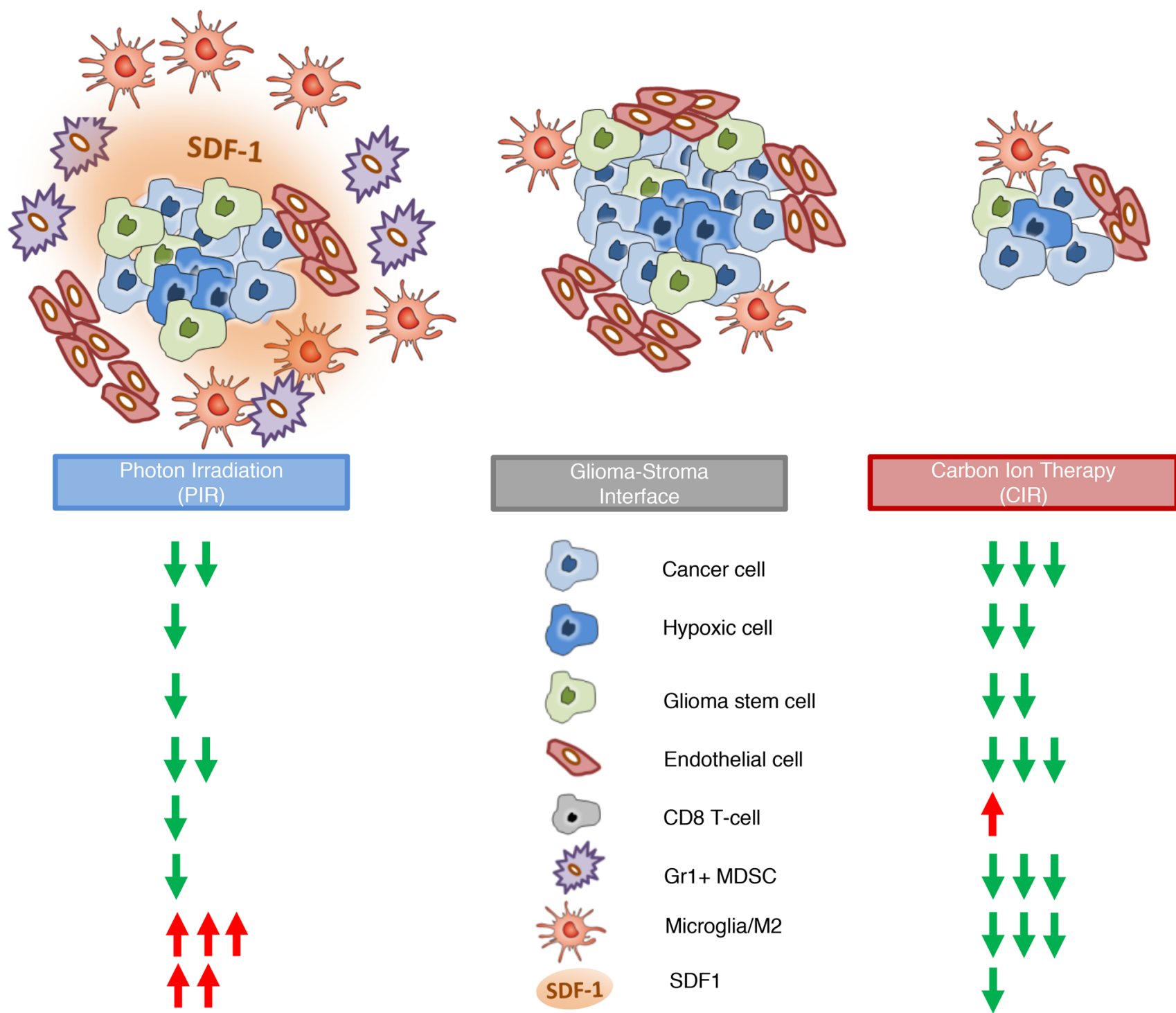

Cancer cell

Hypoxic cell

Glioma stem cell
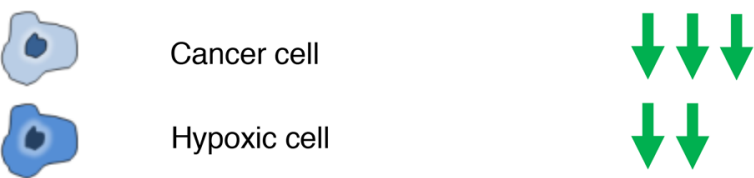

$\downarrow \downarrow$
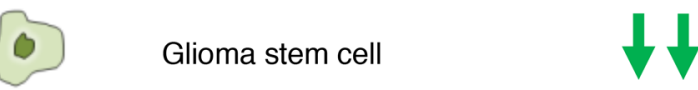

Endothelial cell

CD8 T-cell

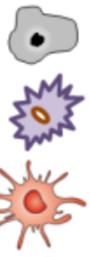

SDF-1

Gr1+ MDSC

Microglia/M2

SDF1

Figure 6. Radiotherapy effects at the tumor-stroma interface. A schematic presentation of the proposed model for cellular and molecular effects of CIR versus PIR based on data reported in this study is shown. Due to their distinct radiobiological effects, CIR is capable of eradicating therapy-resistant tumor subpopulations, e.g., GSCs and hypoxic tumor cells. This observation contrasted with conventional PIR, where these subpopulations were enriched by the therapyinduced selection pressure. Consequently, the concentration of tumor stroma- modulating factors, such as SDF1 released by hypoxic cells, was decreased after CIR, leading to reduced influx of bone marrow-derived immunosuppressive cells (MdSCs) and a less M2-polarized microglia/TAM microenvironment.

or possibly curative treatment. Indeed, experimental approaches to convert the M2 polarization and the immunosuppressive glioma niche have recently shown impressive synergies with immune checkpoint inhibitors (28). The beneficial effects of CIR reported here suggest the need for further evaluation of this promising therapy in multimodal strategies aiming to enhance glioma immunity.

\section{Methods}

Cells and reagents. SMA-560 and G1261 murine glioma cell lines, human primary GSC-enriched cultures (i.e., NCH644, NCH441, and T325), as well as embryonic stem cell-derived microglia were cultured at $37^{\circ} \mathrm{C}$ and $5 \% \mathrm{CO}_{2}(16,29)$. For details, see Supplemental Methods (SM), available online with this article.

Time-lapse microscopy-based wound and coculture migration assay. SMA-560 and G1261 cells were grown to confluence in 96-well ImageLock plates (Essen BioScience). Using a 96-pin WoundMaker, precise wounds were created in all wells. After a gentle wash, cells were irradiated with $0 \mathrm{~Gy}$ and then $4 \mathrm{~Gy}$ of photons or carbon ions. Plates were scanned every 2 hours by IncuCyte (Essen BioScience). Wound confluence metrics were quantified using the IncuCyte FLR imaging system (Essen BioScience). Coculture transmigration 
assay was performed as previously described with minor modifications (30).

Clonogenic survival. Increasing cell numbers (300-7000) were plated in $25-\mathrm{cm}^{2}$ flasks (Sarstedt) and allowed to attach before radiotherapy. Colonies were monitored and counted under a microscope. Anchorage-independent sphere-like colony formation of NCH644 in soft agar was monitored using the CytoSelect 96-well cell transformation assay (Cell Biolabs Inc.) as described previously (10). Sphere-like colonies were counted by microscopy 2 weeks later. In hypoxic experiments, cells were incubated at $1 \% \mathrm{O}_{2} 4$ hours prior to irradiation in a C-Chamber hypoxia chamber (BioSpherix). In the case of combination with TMZ, cells were treated with $10 \mu \mathrm{M} \mathrm{TMZ}$ or vehicle in serum-free medium 2 hours prior to irradiation.

Orthotopic glioma models. To form orthotopic tumors, 1000 SMA-560 Luci+ $^{2}, 2000 \mathrm{Gl} 261^{\text {Luci }}, 10,000$ $\mathrm{NCH} 644^{\mathrm{Luci+}}$, and 10,000 NCH441 $1^{\mathrm{Luci+}}$ cells were stereotactically implanted into the right striatum of 6to 8-week-old female VMDk, C57BL/6, and NCr Nude mice, respectively. Mice were randomized into different groups based on bioluminescence detection ( $>50,000$ counts/second). A single-hemisphere, $5 \times 5 \mathrm{~mm}$ region around the cell injection site was irradiated with a lateral photon beam. The contralateral hemisphere and the rest of the body were protected by a lead shield. For HIR and CIR, mice were fixed on custom-designed holder, and the tumor region was irradiated at HIT within a spread-out Bragg peak of $6 \mathrm{~mm}$ at a water equivalent depth of $114 \mathrm{~mm}$ (LET, carbon: 120 kilo-electron volts/ $\mu \mathrm{m}$, proton: 6 kilo-electron volts/ $\mu \mathrm{m})$.

In vivo bioluminescence imaging. Mice were anesthetized with isofluorane and injected intraperitoneally with $150 \mu 1$ of $30 \mathrm{mg} / \mathrm{ml}$ D-Luciferin (Caliper Life Sciences) for 10 minutes. Afterward, mice were sequentially imaged using the IVIS 100 system coupled with Living Image acquisition and analysis software (Caliper Life Sciences). Bioluminescence counts were calculated within assigned region of interest.

Noninvasive imaging modalities. Mouse MRI was performed using a custom-developed transmit/ receive small animal coil in a conventional whole-body 1.5-T MRI scanner (Symphony, Siemens). Clinical MRI was performed on a 3-T MRI scanner (Tim Trio, Siemens). Tumor metabolism was studied using an in-house-developed FET amino acid tracer. For organ distribution, NCH644 animals were injected 2 weeks after irradiation using in-house-labeled $\mathrm{I}^{131} \alpha$-CD133. Further details are provided in SM.

Immunofluorescence. Sections were fixed either in acetone or $4 \%$ paraformaldehyde (PFA)/PBS, washed, blocked in $1 \% \mathrm{BSA} / \mathrm{PBS}$, and incubated overnight at $4^{\circ} \mathrm{C}$ in 1:50 anti-CD31 550274 (BD Biosciences), 1:100 anti-CD11b ab8878 (Abcam), 1:100 anti-Gr1 ab25377 (Abcam), 1:100 anti-SDF1 sc-6193, or 1:100 anti-CD133/1 (AC133) (Miltenyi Biotec, BergischGladbach). Slides were washed and signals detected with secondary antibodies (1:400) coupled with Alexa Fluor 488 or Alexa Fluor 555 (Invitrogen). Sections were mounted with Fluoromount-G (Southern Biotech). DAPI dye was used to stain nuclei. Microscopy was performed using Zeiss Cell Observer SD.

Transcriptome analysis. Expression profiling using microarray technology and real-time quantitative reverse transcription PCR was performed as described previously (24). TaqMan chemistry and mouse sentrix-8 chips (Illumina Inc) were used (for details see SM). All gene expression profiles are available online at ArrayExpress (http://www.ebi.ac.uk/arrayexpress) under accession number E-MTAB-6252.

Bioinformatics. ANOVA was performed to filter top significant differentially regulated genes at $P<0.01$ distinguishing photon, proton, and carbon groups. GeneGo software (Thomson Reuters) and the Kyoto Encyclopedia of Genes and Genomes database were applied to appraise biological pathways involving candidate genes. Human and murine genes were matched using the Gene Expression Database (http:// www.informatics.jax.org). To enumerate the abundance of 22 immune cell types in the tumor stroma of both SMA-560 and G1261 murine models, CIBERSORT was applied (https://cibersort.stanford.edu/).

Statistics. Statistical analysis of data was computed with the Sigma Plot software package (Systat Software Inc). Statistical analysis of Kaplan-Meier survival estimates was computed with the Statistical Utility for Micro array and Omics data software package (http://angiogenesis.dkfz.de/oncoexpress/software/index.htm). Data are presented as mean \pm SEM unless otherwise noted. Statistical significance was assessed using Student's $t$ test unless otherwise noted. $P<0.05$ was considered statistically significant. All statistical tests were 2-tailed.

Study approval. Animal work was performed according to rules approved by the local and governmental animal care committee instituted by the German government (Regierungspraesidium, Karlsruhe).

\section{Author contributions}

SC, ZT, DL, ODW, UH, JD, CHM, WW, and AA conceived the study. SC, ZT, and DL performed the animal experiments with help for particle irradiation (MM and SB), PIR (SA), radiolabeling of antibodies 
(WM), and PET imaging (SM and $\mathrm{UH}$ ), as well as MRI (MB). In vitro assays were performed by SC, ZT, and ID with help for microglia transwell experiments (PS) and GSC cultures (RW and CR). Data analysis was performed by SC and ZT with help for omics analysis (MK) and interpretation (SC, WW, and AA). The manuscript was mainly written by SC and AA and was critically revised by all authors.

\section{Acknowledgments}

This work was supported by German Krebshilfe (Deutsche Krebshilfe, Max-Eder 108876). It was also in part supported by the German Research Council (DFG, KFO-214), the Charitable Hertie Foundation, and intramural grants of the National Center for Tumor Diseases (NCT3.0_2015.21/22 NCT-PRO and Biodose programs). The funders had no role in the study design, data collection and analysis, decision to publish, or preparation of the manuscript.

Address correspondence to: Sara Chiblak or Amir Abdollahi, Division of Molecular \& Translational Radiation Oncology, Heidelberg Ion Therapy Center, Heidelberg University Medical School, Im Neuenheimer Feld 450, 69120 Heidelberg, Germany. Phone: 49.6221.420; Email: s.chiblak@dkfz.de (SC). Phone: 49.6221.56.39604; Email: a.amir@dkfz.de(AA).

1. Debus J, Abdollahi A. For the next trick: new discoveries in radiobiology applied to glioblastoma. Am Soc Clin Oncol Educ Book 2014:e95-e99.

2. Stupp R, et al. Radiotherapy plus concomitant and adjuvant temozolomide for glioblastoma. N Engl J Med. 2005;352(10):987-996.

3. Stupp R, et al. Effects of radiotherapy with concomitant and adjuvant temozolomide versus radiotherapy alone on survival in glioblastoma in a randomised phase III study: 5-year analysis of the EORTC-NCIC trial. Lancet Oncol. 2009;10(5):459-466.

4. Wick W, et al. MGMT testing — the challenges for biomarker-based glioma treatment. Nat Rev Neurol. 2014;10(7):372-385.

5. Abdollahi A, Folkman J. Evading tumor evasion: current concepts and perspectives of anti-angiogenic cancer therapy. Drug Resist Updat. 2010;13(1-2):16-28.

6. Durante M, Orecchia R, Loeffler JS. Charged-particle therapy in cancer: clinical uses and future perspectives. Nat Rev Clin Oncol. 2017;14(8):483-495.

7. Howard SP, Krauze A, Chan MD, Tsien C, Tomé WA. The evolving role for re-irradiation in the management of recurrent grade 4 glioma. J Neurooncol. 2017;134(3):523-530.

8. Combs SE, et al. Randomised phase I/II study to evaluate carbon ion radiotherapy versus fractionated stereotactic radiotherapy in patients with recurrent or progressive gliomas: the CINDERELLA trial. BMC Cancer. 2010;10:533.

9. Bao S, et al. Glioma stem cells promote radioresistance by preferential activation of the DNA damage response. Nature. 2006;444(7120):756-760.

10. Chiblak S, et al. Radiosensitivity of patient-derived glioma stem cell 3-dimensional cultures to photon, proton, and carbon irradiation. Int J Radiat Oncol Biol Phys. 2016;95(1):112-119.

11. Osswald M, et al. Brain tumour cells interconnect to a functional and resistant network. Nature. 2015;528(7580):93-98.

12. Geisenberger C, et al. Molecular profiling of long-term survivors identifies a subgroup of glioblastoma characterized by chromosome 19/20 co-gain. Acta Neuropathol. 2015;130(3):419-434.

13. Rapp C, et al. Identification of $\mathrm{T}$ cell target antigens in glioblastoma stem-like cells using an integrated proteomics-based approach in patient specimens. Acta Neuropathol. 2017;134(2):297-316.

14. Ferrandon S, et al. Cellular and molecular portrait of eleven human glioblastoma cell lines under photon and carbon ion irradiation. Cancer Lett. 2015;360(1):10-16.

15. Rieken S, et al. Carbon ion irradiation inhibits glioma cell migration through downregulation of integrin expression. Int J Radiat Oncol Biol Phys. 2012;83(1):394-399.

16. Lemke D, et al. Primary glioblastoma cultures: can profiling of stem cell markers predict radiotherapy sensitivity? J Neurochem. 2014;131(2):251-264

17. Zagzag D, et al. Hypoxia-inducible factor 1 and VEGF upregulate CXCR4 in glioblastoma: implications for angiogenesis and glioma cell invasion. Lab Invest. 2006;86(12):1221-1232.

18. Debus C, et al. Feasibility and robustness of dynamic 18F-FET PET based tracer kinetic models applied to patients with recurrent high-grade glioma prior to carbon ion irradiation. Sci Rep. 2018;8(1):14760.

19. Debus C, et al. Impact of 18F-FET PET on target volume definition and tumor progression of recurrent high grade glioma treated with carbon-ion radiotherapy. Sci Rep. 2018;8(1):7201.

20. Dokic I, et al. Next generation multi-scale biophysical characterization of high precision cancer particle radiotherapy using clinical proton, helium-, carbon- and oxygen ion beams. Oncotarget. 2016;7(35):56676-56689.

21. Klein C, et al. Overcoming hypoxia-induced tumor radioresistance in non-small cell lung cancer by targeting DNA-dependent protein kinase in combination with carbon ion irradiation. Radiat Oncol. 2017;12(1):208.

22. Pignalosa D, Durante M. Overcoming resistance of cancer stem cells. Lancet Oncol. 2012;13(5):e187-e188.

23. Wang Q, et al. Tumor evolution of glioma-intrinsic gene expression subtypes associates with immunological changes in the microenvironment. Cancer Cell. 2018;33(1):152.

24. Almog N, et al. Consensus micro RNAs governing the switch of dormant tumors to the fast-growing angiogenic phenotype PLoS One. 2012;7(8):e44001

25. Kioi M, Vogel H, Schultz G, Hoffman RM, Harsh GR, Brown JM. Inhibition of vasculogenesis, but not angiogenesis, prevents 
the recurrence of glioblastoma after irradiation in mice. J Clin Invest. 2010;120(3):694-705.

26. Müller S, et al. Single-cell profiling of human gliomas reveals macrophage ontogeny as a basis for regional differences in macrophage activation in the tumor microenvironment. Genome Biol. 2017;18(1):234

27. Otvos B, et al. Cancer stem cell-secreted macrophage migration inhibitory factor stimulates myeloid derived suppressor cell function and facilitates glioblastoma immune evasion. Stem Cells. 2016;34(8):2026-2039.

28. Saha D, Martuza RL, Rabkin SD. Macrophage polarization contributes to glioblastoma eradication by combination immunovirotherapy and immune checkpoint blockade. Cancer Cell. 2017;32(2):253-267.e5.

29. Campos B, et al. Aberrant self-renewal and quiescence contribute to the aggressiveness of glioblastoma. J Pathol. 2014;234(1):23-33.

30. Abdollahi A, et al. Inhibition of platelet-derived growth factor signaling attenuates pulmonary fibrosis. J Exp Med. 2005;201(6):925-935. 